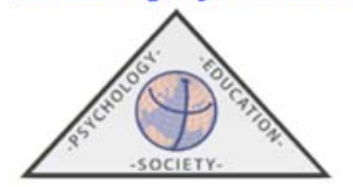

\title{
Niveles de estrés de la población estudiantil en Costa Rica. Diferencias en función de las variables nivel socioeconómico, rendimiento académico, nivel académico y zona geográfica
}

\author{
Sebastien BELHUMEUR*, Alexander BARRIENTOS SEGURA y \\ Axel P. RETANA-SALAZAR \\ Universidad de Costa Rica, Costa Rica
}

(Received on March 2, 2015; Accepted on October 10, 2015)

RESUMEN: En este trabajo se describen los niveles de estrés de la población estudiantil en función de las variables: nivel socioeconómico, rendimiento académico, nivel académico, zona geográfica y género. Para este estudio se aplicó una encuesta a una muestra aleatoria de 112 estudiantes de diferentes carreras de la sede central de la Universidad de Costa Rica. Los resultados mostraron que las mayores fuentes de estrés en los estudiantes son la académica y la financiera. También los estudiantes cuyo lugar de procedencia es diferente al de la sede central de la universidad, presentan mayores niveles de estrés. Los resultados mostraron que las mujeres tienden a manejar mayores niveles de estrés que los hombres.

Palabras clave: Estrés, estudiantes universitarios, Costa Rica.

Stress levels of student population in Costa Rica. Differences according to socioeconomic, academic achievement, educational level, and geographic location variables

\begin{abstract}
In this paper are described the stress levels of the student population about the variables: socioeconomic status, academic performance, academic level, geographical area and gender. For this purpose, a survey was administered to a randomized sample of 112 students of different careers from the main campus of the University of Costa Rica. Results showed that the major sources of stress in students are academic and financial pressures. Also, results showed that students whose place of origin is far from the main campus of the university have higher levels of stress and finally, women tend to handle higher levels of stress than men.
\end{abstract}

Keywords: Stress; university students; Costa Rica.

Correspondencia: Axel Retana-Salazar. Escuela de Nutrición, Facultad de Medicina, Ciudad de la Investigación, Universidad de Costa Rica 2060. E-mail: axel.retana@ucr.ac.cr. 


\section{Introducción}

El estrés se puede comprender como una respuesta a una amenaza directa relacionada con la supervivencia del individuo y la adaptación a estos problemas (Morales 1991). Algunos autores le confieren un particular énfasis al Modelo de Estrés Transaccional de Lazarus, el cual explica que el estrés es un fenómeno derivado de la interacción de estresores del ambiente, procesos fisiológicos, procesos cognitivos y mecanismos de comportamiento (Morales, 1991; Guttman, 1999). La valoración está formada por la combinación de las creencias de las personas, las expectativas, la educación, las experiencias pasadas y la responsabilidad. Los estresores son muy variados y cualquier evento puede verse como un estresor (González de Rivera, 1991).

Considerando al estrés como una reacción adaptativa del organismo ante las demandas de su medio (Guttman, 1999). Todos los organismos experimentan reacciones de estrés durante su vida, pero cuando la reacción del sujeto se prolonga puede agotar las reservas del individuo y traducirse en una serie de problemas fisiológicos (Brosschot et al., 2006; Guttman, 1999).

Para una institución educativa es importante conocer los niveles de estrés académico en sus estudiantes, ya que el estrés se ha asociado a la depresión, enfermedades crónicas, enfermedades cardiacas, fallas en el sistema inmune, distorsiona el ciclo normal del sueño, contribuye al desarrollo de disfunciones sexuales, produce una sensación general de insatisfacción y en algunos casos provoca el completo fracaso universitario y se convierte en un desempeño académico pobre del estudiante(Chávez et al., 2011).

Un cambio de modo de vida abrupto se caracteriza por convertir en inservible muchos repertorios adaptivos que el individuo ha aprendido en situaciones de vida anteriores. Esta situación puede dar como resultado que el individuo se encuentre sometido a una serie de demandas ya sea en el ámbito laboral o educativo, como en sus relaciones interpersonales con las cuales no puede lidiar adecuadamente, pero que a la vez le es imprescindible hacerlo para sobrevivir dentro de la situación novedosa.

Se han llevado a cabo diversos trabajos donde se ha demostrado que los estudios universitarios son el punto culminante del estrés académico. En este nivel se cuenta con un incremento en las cargas de trabajo, pero también coincide con una etapa de la vida en la que el estudiante debe enfrentarse a muchos cambios. Específicamente, el ingreso a la universidad coincide con el proceso de separación de la familia, la incorporación al mercado laboral y la adaptación a un medio poco habitual (Cárdenas y Silva, 2005).

La universidad ha introducido efectos potencialmente provocadores de estrés, de ellos sin duda algunos de los más relevantes, son la competitividad, el mercado laboral, exigencias académicas, exceso de responsabilidad, constantes evaluaciones, exposiciones y realización de trabajos, así como presiones familiares, económicas y sociales que sin darse cuenta provocan un grado de estrés importante el cual se manifiesta generalmente como resultado de las frustraciones originadas por necesidades insatisfechas (Benavente et al., 2010).

En Costa Rica, teniendo uno de los sistemas educativos de mayor desarrollo en América Latina, ubicado junto con Argentina y Uruguay entre los países de modernización temprana con una maciza cobertura escolar, se presentan altos índices de alfabetización y de instrucción universitaria (Weinstein, 1998).Después de revisar las principales bases de datos en busca de trabajos al respecto en Costa Rica (SCOPUS, WoK, Scielo, Latindex, Redalyc, Dialnet, DOAJ, 
BioMed Central) no se han encontrado estudios acerca del estrés en estudiantes universitarios en Costa Rica. Este estudio es pionero en Costa Rica y pretende plantear un primer acercamiento a esta problemática en el país. Por esta razón, este estudiopretende analizar la relación que existe entre las variables nivel socioeconómico, rendimiento académico, nivel académico, zona geográfica y género que se han estudiado en grupos de estudiantes en otros países de América Latina (Marty et al. 2005) las cuales se incluyen en los trabajos de Potter (1991), y el nivel de estrés de estudiantes de la Universidad de Costa Rica. De esta forma, empezar con estos estudios nos permite comenzar un proceso de comprensión sobre las causas del estrés mencionadas anteriormente.

Por lo tanto, el objetivo primario es identificar en un momento determinado, los niveles de estrés de la población estudiantil en función de las variables:nivel socioeconómico, rendimiento académico, nivel académico, zona geográfica y género.

\section{Método}

La metodología en la que se sustenta esta investigación es descriptiva, correlacional y transversal (Valenzuela-Pablos y Fraijo-Sing 2010). El diseño es un estudio aleatorio de prevalencia de estrés en estudiantes de laUniversidad de Costa Rica.

Participantes

En noviembre del 2011 se realizó una encuesta de participación voluntaria sobre niveles de estrés y condición socio económica a 112 estudiantes de la sede central de la Universidad de Costa Rica. Las carreras consideradas fueron ingeniería, ciencias económicas y ciencias sociales. Participaron estudiantes de todos los niveles de carrera. Se comparó entre sí las diferencias en el nivel de estrés en función de variables como nivel socioeconómico, rendimiento académico, nivel académico, zona geográfica y género. Estas variables fueron consideradas teniendo en cuenta que han sido indicadas como posibles fuentes o antecedentes del estrés. La distribución por sexo de los encuestados fue de 43 mujeres y 69 hombres. El ámbito de edad fue de 18hasta 31 años cumplidos. La población estudiada presentó un promedio de 23 años con una desviación de \pm 1,3.

\section{Procedimiento}

Los integrantes de la población de estudio se seleccionaron al azar, y aunque pertenecen a varias carreras en tres Facultades, la propuesta metodológica seguida es la implementación de un sistema de muestreo aleatorio, donde los individuos son seleccionados al azar del total de la población. Se trabaja con un segmento etario de la población de estudiantes analizada.

\section{Instrumentos}

Para obtener la información acerca del grado de estrés se usó un cuestionario Estrés y rendimiento en el trabajo (Marty et al. 2005). El instrumento ha sido referido en trabajos anteriores en el estudio de las relaciones del estrés con el rendimiento (Potter 1991, Marty et al. 2005), el cuál consta de 20 preguntas. La herramienta utilizada cuenta con preguntas que pueden tener injerencia en el desarrollo del estrés. Dentro de las distintas preguntas, está lo que es la caracterización de la condición sociodemográfica donde se incluyen preguntas tales como "Lugar de residencia de su grupo familiar" en el que se le dan opciones por provincias para la 
escogencia de la respuesta. En este mismo sentido se valora si los estudiantes durante el ciclo lectivo se mantienen con un grupo familiar o no mediante preguntas como " $¿ V i v e$ usted con un grupo familiar durante el ciclo lectivo?”. También se pueden encontrar preguntas en cuanto a la caracterización de la situación socioeconómica, donde se valoran los factores económicos que pueden tener injerencia en el desarrollo de estrés, donde se presentan preguntas como "Durante el ciclo lectivo paga usted alquiler o no" o "Recibe usted algún tipo de beca durante el ciclo lectivo” y si "Cual es la situación laboral durante el tiempo de estudio” y “¿Cuál es el ingreso promedio?”. Por ultimo, la herramienta cuenta con preguntas de caracterización de la situación personal, donde se valoran otras variables como “¿Cuál es su estado civil?” o "Número de créditos matriculados en este ciclo lectivo”y “¿Cuáles cree usted que son sus principales fuentes de estrés?” lo que debe escoger de una lista de posibles estresores. Estos factores se evaluaron con referencia a síntomas de estrés en los últimos cuatro meses, que había que calificar con un número del 1 al 5 según su intensidad, siendo $1=$ nunca o rara vez, $2=$ ocasionalmente, 3 = a menudo, $4=$ frecuentemente y $5=$ siempre.

Se clasificaron las respuestas según el puntaje siguiente: 20 a 36 puntos = estrés bajo, 37 a 52 puntos= estrés medio y de 53 a 76 puntos = estrés alto. Esta escala es la utilizada en la literatura acerca de estrés en estudiantes (Marty et al. 2005, Caldera-Montes et al. 2007). El motivo por el que se usó como máximo 76 puntos se debe a que la puntuación más alta obtenida fue de 73 puntos. Se consignaron además datos socio-demográficos y un ítem sobre la(s) principal(es) fuente(s) de estrés que más le afectaba.Se consignaron además datos sociodemográficos, para lo cual se elaboró una encuesta y los resultados se tomaron según una escala presentada de nivel socioeconómico según el rango salarial de ingresos al hogar.

A pesar de que este instrumento es citado en numerosos trabajos de evaluación del estrés (Potter 1991, Girón-Alvarado 2004, Marty et al. 2005), en ninguno se informa del cálculo de fiabilidad de esta herramienta. No obstante, su amplio uso indica que la herramienta es muy aceptada en la publicación de numerosos trabajos de investigación en estrés. En este trabajo a partir de una matriz de 112x20 analizada mediante el programa para cálculo del alfa de Cronbach publicado por Ledesma (2004) denominado Alpha CI. Los resultados indican un valor del alfa para el instrumento utilizado de 0,71 y las desviaciones típicas de los ítems oscilan entre 0,480 y 0,802 , por lo que los valores de las variaciones no se hayan altamente desviados entre sí. Por otra parte, los valores de las varianzas de los ítems osciló entre 0,380,81 por lo que no fue necesario desestimar ni reformular ningún ítem según los establecen los límites de confianza de la prueba. Por otra parte, la literatura establece que los valores del alfa de Cronbcah por encima de 0,70 se consideran fiables en estudios de investigación exploratoria (Huh et al 2006, Bojórquez-Molina et al. 2013).

\section{Resultados y discusión}

De los encuestados (Cuadro 1), se observa que el mayor porcentaje de universitarios consideran que su mayor fuente de estrés es académica (87\%), seguida por la financiera (46\%) y presiones familiares (18\%). Otros factores tomados en cuenta oscilan entre el 6-8\% por lo que parecen ser los de menor peso entre las causas de estrés en la población estudiada. 
Tabla 1. Fuente de estrés de los estudiantes encuestados de la Universidad de Costa Rica.

\section{Fuentes de estrés Porcentaje de personas}

\begin{tabular}{lc}
\hline Académico & $87 \%$ \\
\hline Financiero & $46 \%$ \\
\hline Ausencia de vida social & $7 \%$ \\
\hline Presiones Familiares & $18 \%$ \\
\hline Salud & $6 \%$ \\
\hline Reubicación Geográfica & $6 \%$ \\
\hline Otras & $8 \%$
\end{tabular}

Esto se debe a que la competitividad actual en la cual los estudiantes son constantemente evaluados para superar niveles de educación, provoca estados de tensión y frustración por las exigencias académicas en su preparación (Chávez et al. 2011). La proximidad y fracaso experimentado en los exámenes (Muñoz-García y León-Rubio 1990), la sobrecarga curricular, ausencia de pausas entre clases, exceso de responsabilidades, tiempo limitado para hacer trabajos, sumado a esto existen otros eventos como los factores sociales, disgusto con los miembros de la familia y amigos, malas relaciones interpersonales, conflictos religiosos, entre otros (Feldman et al. 2008). Esto se puede ver agravado por factores de la dinámica familiar, como puede ser el divorcio de los padres y exceso de exigencias de los mismos (Serra-Taylor 2010). Otro componente es el económico como insuficiente ingresos familiares o del propio estudiante si trabaja (Serra-Taylor 2010). Finalmente, influyen también aspectos personales como la baja autoestima, cambio en los patrones de vida, el explorar reiteradamente múltiples áreas de estudio y trabajo (Benavente et al, 2010, Serra-Taylor 2010).

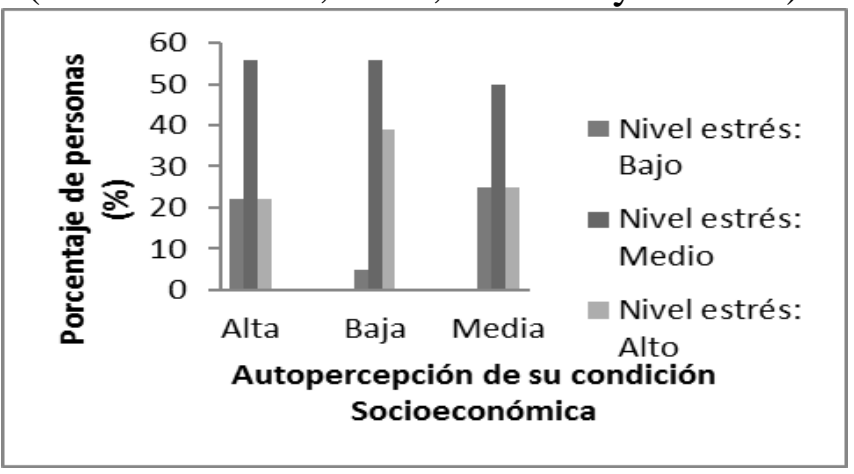

Figura 1. Distribución del nivel de estrés en función de la autopercepción socioeconómica en estudiantes de la UCR. 
La figura 1 muestra que los estudiantes que se perciben con una condición socioeconómica media y alta, mantienen una distribución del estrés similar, a diferencia de los estudiantes que se perciben con una condición económica baja, para quienesel nivel de estrés alto informado es más frecuente.

En la figura 2 se aprecia que el nivel de estrés alto en estudiantes que han tenido problemas financieros en los últimos cuatro meses tiende a incrementarse comparado con los estudiantes que no han tenido problemas financieros recientes, sin embargo los demás niveles de estrés considerados (bajo y medio) no muestran variaciones sensibles. Según Benavente y colaboradores (2010), los problemas económicos presentados por algunos estudiantes incrementan significativamente el nivel de estrés. Las estadísticas llevadas a cabo en Costa Rica por el Ministerio de Educación Pública no incluyen a las universidades por lo que no hay datos acerca de los efectos en esta población por la deserción y los problemas económicos que pueden ayudar a gestarla. El Informe del Estado de la Nación (CONARE, 2014) ha revelado después de 20 años de estudios que Costa Rica es uno de los países más caros de América Latina.

Lo anterior se evidencia en que las universidades públicas como la Universidad de Costa Rica con horarios que no permiten al estudiante mantener un trabajo, está afectando el que solo cierta clase social pueda acceder a la educación pública. El reflejo de que para un $46 \%$ de los encuestados el factor económico sea motivo de preocupación puede obedecer entre otras razones a la dificultad para poder mantenerse en la universidad sin poder trabajar, lo que puede ser parte del problema que refleja un $18 \%$ de los encuestados acerca de problemas con la familia.

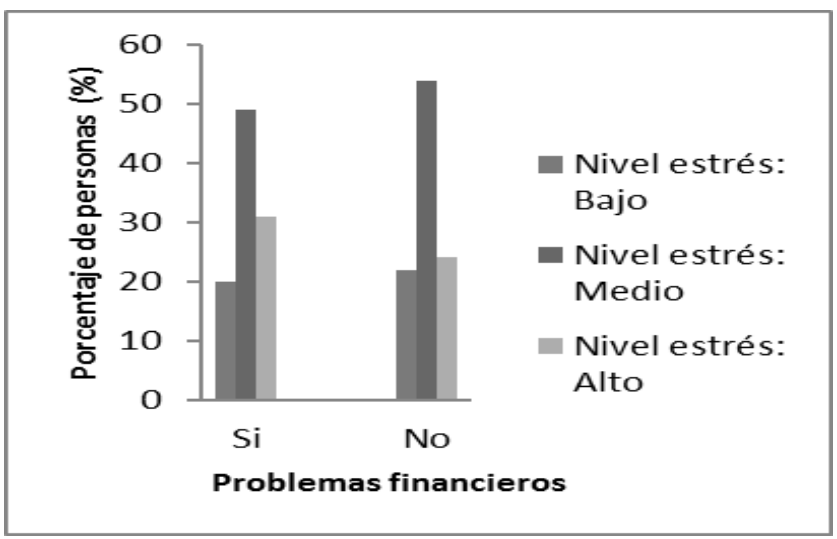

Figura 2. Distribución del nivel de estrés en función de problemas financieros en los últimos cuatro meses.

Otro dato importante que se puede deducir de los datos es que el estrés es manejado de manera diferente según el género (Fig. 3). En la población analizada se evidencia que hay una distribución muy homogénea de los niveles de estrés por parte de la mujeres en donde tanto el nivel de estrés bajo, como medio y alto muestran valores superiores al 30\% y menores al $40 \%$ respectivamente, mientras que en los varones los niveles de estrés alto y bajo presentan niveles por debajo del $20 \%$ en el primer caso y ligeramente por encima en el segundo, mientras que el nivel de estrés medio llega a niveles superiores al 60\%. Estos datos parecen indicar que hay un mejor manejo del estrés por parte de la población masculina, lo que coincide con los datos 
obtenidos por Chávez y colaboradores (2011), ya que en su estudio, las mujeres presentan niveles de estrés superiores a los hombres y esto se debe sobre todo a los estilos de afrontamiento de ambos sexos. En el caso de la Universidad de Costa Rica parece ser que más bien se trata de una diferente proporción de la distribución del estrés.

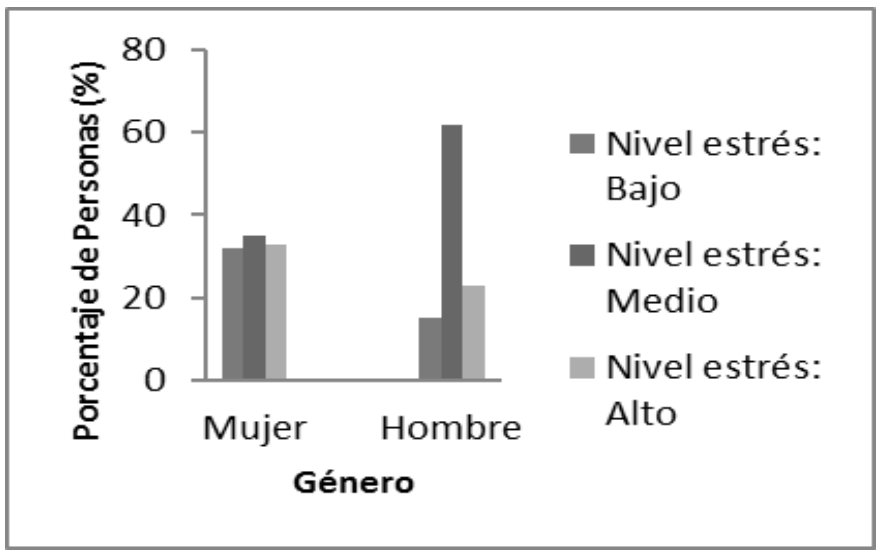

Figura 3. Distribución del nivel de estrés en función del género de los estudiantes universitarios.

De acuerdo con los datos arrojados por la encuesta (figura 4) se observa que el nivel más alto de estrés se encuentra en los estudiantes que son de otra zona lejos de la sede universitaria. Lo cual según Renk\&Creasey (2003), mencionado por Chávez et al. (2011), la tendencia general indica que los estudiantes que viven fuera de la familia (amigos o solos), podrían tener niveles de estrés más altos que aquellos que viven con ella. En este caso, de nuevo la procedencia se convierte en un problema más que se suma a los problemas económicos debido a que la Universidad como institución no tiene la capacidad de darle hospedaje a sus estudiantes de zonas rurales, por lo que estos deben de pagar el servicio de hospedaje, lo que incrementa el problema económico que se suma a la dependencia de la familia por parte del estudiante.

El Informe del Estado de la Nación (CONARE,2014) muestra que la pobreza está afectando la escolaridad y que en el segmento de edades entre 20-24 años Costa Rica cuenta con porcentajes muy por debajo de los de Chile, Ecuador y Venezuela en cantidad de personas con educación secundaria completa, lo que reduce la cantidad de estudiantes que pueden acceder a la educación superior.

Este mismo documento muestra que los años de estudio se han incrementado lo que aumenta el estrés académico de los estudiantes que necesitan integrarse a la fuerza laboral y no les es posible, haciendo cada vez más complejo el panorama de la educación superior en Costa Rica. Esto genera presiones económicas y familiares sobre los estudiantes.

Al comparar el nivel de estrés y el año de carrera en que se encuentran los estudiantes (figura 5) se observa que en tercer año es donde existe el mayor porcentaje de personas con nivel de estrés alto. En general, en todos los años de la carrera los estudiantes presentan un nivel de estrés medio. Esto se debe posiblemente a que la distribución en la carga de trabajo en el diseño curricular de la institución no es homogénea a lo largo de los diferentes semestres (Chávez et al., 2011).Se podría decir que, además de las cargas tanto económica como social que generan ya cierta presión en los estudiantes, también el entorno y la presión por permanecer en la Universidad sin contar con ingresos económicos propios en la mayoría de los 
casos suma en cuanto a la presión. Además, está también la demanda de esfuerzo en horasestudio por parte de los cursos se incrementa a cómo avanza la carrera, sin que haya una repartición más homogénea de los cursos según su dificultad lo que aumenta la probabilidad de perder cursos, haciendo que se alargue la carrera.

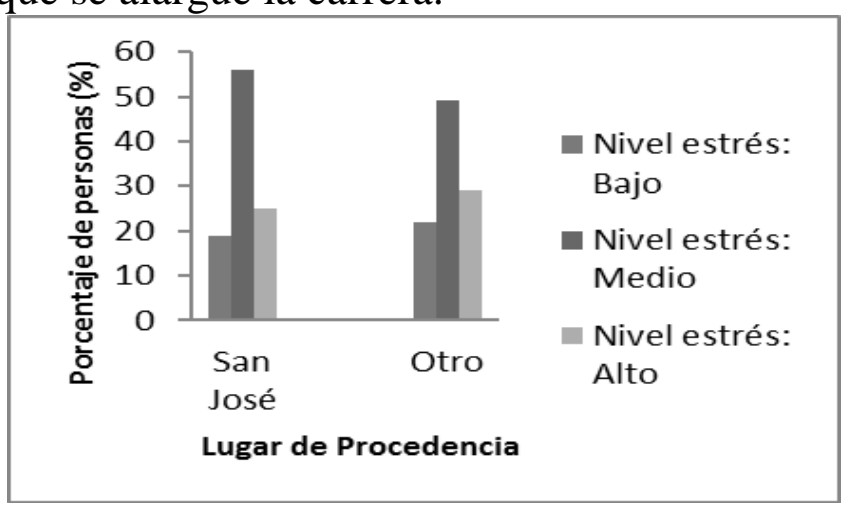

Figura 4. Distribución del nivel de estrés en función el lugar de procedencia de los estudiantes universitarios.

Llevar a cabo estudios de este tipo en instituciones como la Universidad de Costa Rica es realmente importante, ya que muchos estudios realizados en otros países indican que un gran porcentaje de los estudiantes han llegado a considerar la posibilidad de desertar de la universidad debido al estrés académico. Por esto, resulta importante ayudar al alumno a no perder su proyecto universitario por este motivo.

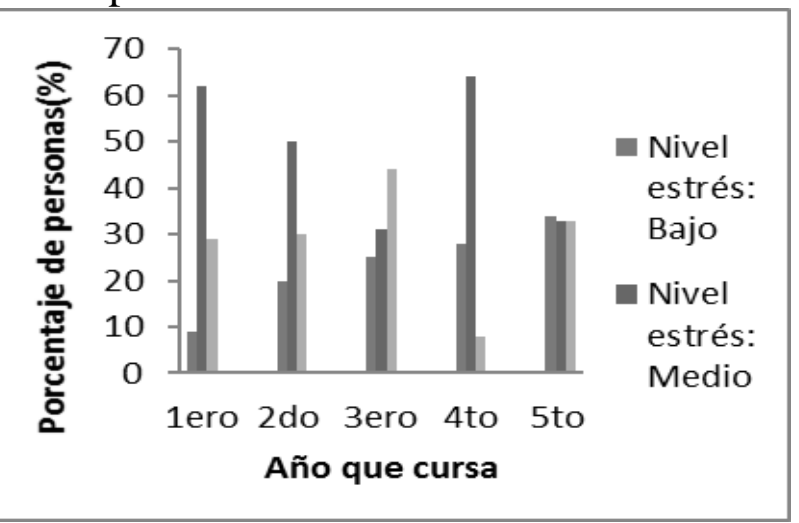

Figura 5. Distribución del nivel de estrés en función al nivel académico de los estudiantes universitarios.

Como se desprende de los resultados hay un incremento del estrés debido a múltiples motivos, entre ellos la presión académica. Esto se refleja en un incremento en el tiempo de estudio requerido para concluir los estudios de una carrera, este aumento de casi dos años se ha obtenido a partir de los datos tomados entre los años 1993-2013 (CONARE 2014). Esto se aúna al hecho de que la Universidad de Costa Rica cuenta con una oferta de horarios diurna en su mayoría lo que hace que sea difícil trabajar y estudiar. Entre las medidas que debería tomar la institución se hallan: a) incrementar el número de docentes, b) ofrecer una mayor variedad de horarios de estudio que faciliten al estudiante la subsistencia y c) adaptar los programas de las carreras para que se cumplan en el periodo establecido en los programas de estudio de las carreras. 
Agradecimientos.

Al Centro de Investigación en Estructuras Microscópicas (CIEMIC) por el apoyo en la realización de este estudio como la Vicerrectoría de Investigación por facilitar recursos para el desarrollo del mismo y al Programa Universitario de Biología Aplicada (PUA) del CIEMIC por su apoyo. De igual forma se le agradece a la Escuela de Psicología de la Universidad de Costa Rica, por facilitar el espacio para el desarrollo de estas iniciativas de investigación. A los señores Gary Fernández, Katerina Fernández, Yerlin Pereira, Edgar Pacheco por colaborar en la toma de datos para este trabajo. Se le agradece además a los revisores anónimos que con sus comentarios han ayudado a mejorar este trabajo.

\section{Referencias}

Benavente, T., Callata, L. y Quispe, P. (2010). Nivel de estrés y estrategias de aprontamiento en estudiantes de la facultad de enfermería UNAS Arequipa 2006. Revista Enfermería Global, 4,5-21.

Bernal, R., Herrera, R. y Rincón, R. (1992).Estrés y enfermedad en la iniciación de la vida universitaria. Revista Colombiana de Psicología,8,75-84.

Blouin, M. y Bergeron, C. (1997). Dictionnaire de la réadaptation, tome 2: termes d'intervention et d'aidestechniques. Les Publications du Québec, 37. Recuperado dehttp://www.med.univ-rennes1.fr/iidris/cache/es/23/2330

Bojórquez Molina, J.A., López Aranda, L., Hernández Flores, M.E. y Jiménez López, E. (2013). Utilización del alfa de Cronbach para validar la confiabilidad de un instrumento de medición de satisfacción del estudiante en el uso del software Minitab. Eleventh LACCEI Latin American and Caribbean Conference for Engineering and Technology (LACCEI'2013)"Innovation in Engineering, Technology and Education for Competitiveness and Prosperity” August 14 - 16, 2013 Cancun, Mexico.1-9.

Brosschot,J. F., Gerin,W yThayer, J. F. (2006). The perseverative cognition hypothesis: a review of worry, prolonged stress-related physiological activation, and health. Journal of Psychosomatic Research,60,113-124.

Caldera-Montes, J.F., Pulido-Castro, B.E. y Martínez-González, M.G. (2007). Niveles de estrés y rendimiento académico en estudiantes de la carrera de Psicología del Centro Universitario de Los Altos. Revista de Educación y Desarrollo, 7,77-82.

Cárdenas, V. y Silva, I. (2005). El estrés en adolescentes. Tesis. Lic. En Psicología, Universidad Autónoma Metropolitana, Iztapalapa.

Chávez, T., Hidalgo, P., Pulido, M., Serrano, M., Valdés, E. y Vera, F. (2011). Estrés académico en estudiantes universitarios. Revista Psicología y Salud, 21,2-7.

Consejo Nacional de Rectores (CONARE). (2014). Informe del Estado de la Nación en desarrollo humano sostenible. Consejo Nacional de Rectores y Defensoría de los Habitantes. 560pp.

Feldman, L., Goncalves, L., Chacón-Piugnau, G., Zaragoza, J., Bagés, N. y De Pablo, J. (2008). Relaciones entre estrés académico, apoyo social, salud mental y rendimiento académico en estudiantes universitarios venezolanos. UniversitasPsychologica,7,739-751.

Girón Alvarado, A.P. (2004). Elementos estresores que afectan el desempeño laboral en los trabajadores de la empresa municipal de agua de la ciudad de Guatemala Empagua. 
Informe Final de Investigación Presentado al Honorable Consejo Directivo de la Escuela de Ciencias Psicológicas. Universidad de San Carlos de Guatemala, para optar al grado de Licenciatura en Psicología. 86pp.

González de Rivera, J.L. (1991). Factores de estrés y vulnerabilidad a la enfermedad. Monografías de Psiquiatría, 3,1-5.

Guttman, B. (1999). Biology. WCB/McGraw-Hill.

Hernández Z., y Romero E. (2010). Estrés en Personas Mayores y Estudiantes Universitarios: Un Estudio Comparativo. Revista Psicología Iberoamericana, 18,56-68.

Huh, J., Delorme, D.E. \& Reid, L.N. (2006). Perceived Third Person Effects and Consumer Attitudes on Prevetting and Banning DTC Advertising. Journal of Consumer Affairs, 40,90-116.

Ledesma, R. (2004). AlphaCI: un programa de cálculo de intervalos de confianza para el coeficiente alfa de Cronbach. Psico-USF, 9, 31-37.

Marty, C., Lavin, M., Figueroa, M., Larraín de la C, D. y Cruz, M. (2005). Prevalencia de estrés en estudiantes del área de la salud de la Universidad de los Andes y su relación con enfermedades infecciosas. Revista Chilena de Neuro Psiquiatría, 43, 25-32.

Morales, M. (1991). Reacciones y formas de enfrentamiento al estrés en el estudiante de medicina de la Universidad de Costa Rica. Tesis de Maestría en Psicología, Universidad de Costa Rica. San José.

Muñoz-García, F. J. y León-Rubio, J. M. (1990). Exámenes Universitarios y Salud: Un estudio Psicosocial sobre el estrés académico. Revista de Enseñanza Universitaria, 1,49-68.

Potter B. (1991). Estrés y rendimiento en el trabajo: Transforme las presiones del trabajo en productividad. México D.F: Editorial Trillas.

Renk, K. y Creasey, G. (2003).The relationship of gender, gender identity and coping strategies in late adolescents. Journal of Adolescence, 26, 159-168.

Serra-Taylor, J.A. (2010). Aspectos psicosociales y el rendimiento académico en estudiantes universitarios. Cuadernos de Investigación en Educación, 25, 75-89.

Valenzuela Pablos, L.M. y FraijoSing, B.S. (2010). Integración y estrés en estudiantes universitarios. Memorias del XI Congreso Nacional de Investigación Educativa / 16. Sujetos de la Educación / Ponencia.

Weinstein, J. (1998). La búsqueda del tesoro perdido. Educación y juventud en América Latina. En: UNESCO, Boletín $N^{\circ}$ 45, Proyecto Principal de Educación en América Latina y el Caribe.(71-87). Santiago, Chile: Publicaciones OREALC 